\title{
Die Bewegung der Blockfreien: "Größte Friedensbewegung der Geschichte«?
}

\author{
von Volker Matthies
}

\section{Zur Friedenspolitischen Relevanz und Selbsteinschătzung der Blockfreien}

Historisch gesehen entstand die Bewegung der Blockfreien nach dem 2. Weltkrieg als eine "major non-military initiative" "von meist jungen Staaten der Dritten Welt, die ihre Existenz und Unabhängigkeit durch die Politik der Militärblöcke ebenso gefährdet sahen wie durch überkommene Strukturen der Fremdherrschaft, Abhängigkeit, Unterentwicklung und Ungleichheit (u. a. Kolonialismus, Imperialismus). Blockfreiheit war eine Strategie dieser Staaten, ihre Sicherheit entgegen der klassischen Gewohnheit nicht durch militärische Allianzbindungen zu gewährleisten, sondern gerade durch das Gegenteil davon, die Abstinenz von den Machtblöcken. In dieser Hinsicht stellte Blockfreiheit ein alternatives und neues Konzept von Sicherheit dar, das nicht auf der Prämisse der "Macht-Balance" (Allianz- und Abschreckungspolitik), sondern auf der Prämisse "Friedlicher Koexistenz" beruhen sollte. ${ }^{2}$

Allerdings verstand sich die Bewegung der Blockfreien nie als eine pazifistische Kraft; zwar unterstrich die Bewegung immer wieder das Gewaltverbot in der internationalen Politik, doch nahm sie das Recht auf nationale, anti-koloniale Befreiungskriege davon aus, die sie sozusagen zu den "gerechten Kriegen" zählte. ${ }^{3}$ Dem Jugoslawen Komatina zufolge "ist die Blockfreiheit eine 'Friedensbewegung', aber nicht im Sinne einer pazifistischen Bewegung, die nach Frieden 'um jeden Preis`strebt, da dieses bedeuten würde, daß die Idee der Veränderung der internationalen Beziehungen aufgegeben würde und die bestehenden repressiven Strukturen, die die Gleichberechtigung in der Welt gefährden, verewigt würden. ${ }^{4}$

* Der nachfolgende Aufsatz basiert auf Teilen einer längeren Ausarbeitung des Verfassers zum Thema "Blockfreiheit als Sicherheitspolitik", Institut für Allgemeine Uberseeforschung, Diskussionsbeiträge 30, München-Köln-London 1983.

1 Vgl. S. D. Muni, Non-Alignment and the Security Parameter, in: International Studies, vol. 20, nos. 1-2, Januar-Juni 1981, S. 160.

2 Vgl. Vlado Benko, Non-alignment and the Problems of Peace and Security on a General World Scale, in: Non-Alignment in the Eighties, Institute of International Politics and Economics/Review of International Affairs, Belgrad 1982.

3 Vgl. A. W. Singham und Shirley Hune, Principles of Non-alignment, in: U.S. Bajpai (ed.), Non-Alignment. Perspectives and Prospects, New Delhi 1983, S. 4 f.

4 Zitiert nach Miljan Komatina, Blockfreiheit und Sicherheit, in: Internationale Politik, H. 796 vom 5. Juni 1983, S. 14. 
Unter dem Eindruck des verschärften Rüstungswettlaufs und Ost-West-Konflikts widmeten sich die Blockfreien auf ihrer 7. Gipfelkonferenz in New Delhi 1983 intensiv der Friedensfrage. Sie übten scharfe Kritik an den Supermächten (im indischen Entwurf der Schlußerklärung war vom "nuklearen Terrorismus" dieser Mächte die Rede!), verurteilten die Doktrin der Abschreckung und des Mächtegleichgewichts sowie Úberlegungen zur Gewinnbarkeit eines Atomkrieges und solidarisierten sich mit den Friedensbewegungen in den blockgebundenen Ländern. ${ }^{5}$ In ihrer Eröffnungsansprache an die Konferenz bezeichnete Indira Gandhi die Bewegung der Blockfreien gar als die "größte Friedensbewegung der Geschichte ${ }^{6}{ }^{6}$

Dieser Vergleich erscheint jedoch schon insofern fragwürdig, als es sich bei den Friedensbewegungen in Europa und den USA ja um nichtstaatliche, soziale Bewegungen handelt, während die Blockfreien eine (überwiegende - wenngleich auch nicht ausschließliche) Bewegung von Staaten sind. Ferner müßte auch der substantielle Gehalt dieser Behauptung geprüft werden. Wie bei den Friedensbewegungen in Europa und den USA steht dabei das Bemühen der Blockfreien um den "negativen Frieden" (= Abwesenheit von direkter, personaler, physischer Gewalt) ${ }^{7}$ im Mittelpunkt; demgemäß soll nachfolgend die Rolle der Blockfreien im Zusammenhang mit der internationalen Sicherheit bzw. dem "Weltfrieden" (Blockfreiheit und Ost-West-Konflikt/Blockfreiheit und Abrüstung) und der Sicherheit bzw. dem "Hausfrieden" zwischen und in blockfreien Staaten (Kriege und Friedliche Streitbeilegung/Innerer Frieden) untersucht werden. Doch bemühen sich die Blockfreien auch um den "positiven Frieden" (= Abwesenheit von indirekter, struktureller Gewalt), zumindest auf der internationalen Ebene, indem sie sich im Nord-Süd-Bereich für die Schaffung einer "Neuen Weltwirtschaftsordnung" und einer "Kollektiven Self-Reliance" der Dritten Welt einsetzen. In diesen Zusammenhang gehört auch das von den Blockfreien im Rahmen ihrer Abrüstungsphilosophie favorisierte Konzept von "Abrüstung und Entwicklung" (= also Ressourcenfreisetzung aus Abrüstungsmaßnahmen für friedliche Aufbauzwecke) sowie ihr Bestreben, das Konzept "internationaler Sicherheit" über seine konventionelle militärische Bedeutung hinaus um eine ökonomische Komponente zu erweitern. ${ }^{8}$

Im Kontext der "Krise der strategischen Doktrinen der Gegenwart" und der "Suche nach einem neuen Sicherheitsparadigma" ist die Blockfreiheit von der europäischen Friedensforschung und -bewegung sozusagen "neu entdeckt" worden. ${ }^{9}$ Bei den seit einigen Jahren in Europa stattfindenden intensiven Diskussionen über alternative Sicherheitspolitiken, über ein die Blöcke transzendierendes europäisches Sicherheitssy-

5 Vgl. die Schlußerklärung der 7. Gipfelkonferenz, abgedruckt in: Internationale Politik, H. 792 vom 5. April 1983.

6 Zitiert nach Internationale Politik, H. 792 vom 5. April 1983.

7 Zum positiven/negativen Friedensbegriff siehe Johan Galtung, Gewalt, Frieden und Friedensforschung, in: Dieter Senghaas (Hrsg.), Kritische Friedensforschung, Frankfurt/Main 1972, S. 55-104.

8 So auf der 7. Gipfelkonferenz der Blockfreien im März 1983 in New Delhi, Schlußerklärung (Wirtschaftlicher Teil), abgedruckt in Internationale Politik, H. 792 vom 5. April 1983.

9 Vgl. hierzu die Konferenzberichte von Hans Günter Brauch und Eva Senghaas-Knobloch, in: DGFK-Informationen 2/81, S. 42-47. 
stem und über "Atomwaffenfreie Zonen" tauchen Konzepte und Ideen auf, die der Bewegung der Blockfreien "alt-vertraut" sind.

Unter theoretischen Gesichtspunkten könnte den Blockfreien durchaus eine friedensfördernde Funktion zukommen. Das internationale System befindet sich infolge der Ausbreitung und Streuung von ökonomischer und militärischer Macht auf immer mehr Akteure derzeit in einem "Ưbergang von einer duopolistisch bzw. oligopolistisch geprägten Sicherheitsstruktur zu einem multipolaren Weltsystem . . ., in dem die Chancen auf unilaterale Kontrollierbarkeit und Steuerung von Konflikten ständig sinken “. ${ }^{10}$ Während sich in diesem Zusammenhang herkömmliche "individuell-eigennützige Machtstrategien" als zunehmend problematisch erweisen, scheinen hingegen "kollektiv-gemeinnützige Vertrauensstrategien" wie die Blockfreiheit diesem Wandlungsprozeß besser zu entsprechen. ${ }^{11}$

\section{Blockf reiheit und "Weltf rieden"}

\subsection{Die Blockfreien als "Vermittler" und "Friedensstifter" im Ost-West-Konflikt?}

Nach der Analyse der Blockfreien stellten und stellen die politisch-militärischen Machtblöcke die größte Bedrohung für ihre Sicherheit und den Weltfrieden dar. Aus dieser Analyse folgern die Nicht-Beteiligung an den Blöcken sowie der Kampf gegen jede Art von Blockpolitik. Von Beginn an war mit der Ablehnung der Blöcke durch die Blockfreien auch der Anspruch verbunden, zwischen den Blöcken als "Vermittler" und im Ost-West-Konflikt als "Friedenstifter" aufzutreten. In dem Kalkül der "Gründungsväter" der Blockfreienbewegung (vor allem Nehru, Nasser und Tito)

"one had to create in Afro-Asia an "area of peace", whose "mobilization of the moral violence of people" against "situations of strength" and military alliances would introduce an element of systemic flexibility, act as a bridge-builder and a communication channel, and mediate to reduce the level of conflict. Their practical means of action were the consolidation of the universal actor - the U.N. - and strengthening the application of the principles of peaceful coexistence as norms governing the functioning of the international system. ${ }^{12}$

Im Rahmen der Vereinten Nationen haben die Blockfreien zweifellos zur Bekräftigung des Konzepts kollektiver Sicherheit, des Gedankens allgemeiner und vollständiger Abrüstung sowie der Ansätze des internationalen Krisenmanagements beigetragen (Mit-

10 Zitiert nach Gerald Braun, Perspektiven und Modelle globaler Sicherheitspolitik, in: Karl-Ernst Schulz (Hrsg.), Streitkräfte im gesellschaftlichen Wandel, Bonn 1980, S. 372.

11 Vgl. hierzu Volker Matthies, Sicherheitspolitik und Dritte Welt, in: Gegenwartskunde 2/1983; Begrifflichkeit nach Daniel Frei und Peter Gaupp, Das Konzept "Sicherheit"-Theoretische Aspekte, in: Klaus-Dieter Schwarz (Hrsg.), Sicherheitspolitik, 3. neu bearb. Aufl., Bonn 1981.

12 Zitiert nach Bahgat Korany, Social Change, Charisma and International Behaviour: Toward a Theory of Foreign Policy-making in the Third World, Leiden 1976, S. 50. 
arbeit der Blockfreien in Vollversammlung und Sicherheitsrat, bei Friedenssicherungsaktionen; Propagierung von "Zonen des Friedens", seit 1971 besonders für den Indischen Ozean; Initiierung der UN-Sonderkonferenzen über Abrüstung von 1978 und 1982, Abstimmungsverhalten in internationalen Krisensituationen wie z. B. in der Afghanistan-Frage).

Fragwürdiger erscheinen jedoch die Möglichkeiten und Fähigkeiten der Blockfreien, im Bereich der Kernsubstanz des Ost-West-Konflikts überhaupt Vermittlungs- und Lösungsansätze anbieten zu können, ganz abgesehen von der Frage nach der Akzeptanz blockfreier Vermittlung durch die Supermächte selbst. So ist die von den Blockfreien beanspruchte "Friedens-Funktion" im internationalen System nicht nur außerhalb der Bewegung als überzogen oder gar falsch bezeichnet worden, sondern sie wird auch von Forschern aus blockfreien Ländern selbst bestritten. ${ }^{13}$ Das häufigste Argument in diesem Zusammenhang lautet, daß es den Blockfreien zunehmend an der nötigen "Objektivität" und "Unbefangenheit" fehle, die zu den unerläßlichen Qualitäten eines erfolgreichen Vermittlers gehörten. Immer stärker habe sich die Mitgliedschaft der Bewegung in den Ost-West-Konflikt und in die Blockpolitik verstricken lassen, wodurch sie in eine fundamentale "Orientierungskrise" geraten sei, die eine autonome Handlungs- und Vermittlungsfähigkeit in der internationalen Politik weitgehend ausschließe. ${ }^{14}$

Vielmehr mußten sich die Blockfreien zunehmend mit dem Vorwurf auseinandersetzen, sie betrieben direkt oder indirekt das politische Geschäft des einen oder anderen Blocks: Der Westen, in dessen Einschätzung die Blockfreien politisch der Sowjetunion zu nahe waren, forderte von der Bewegung eine Position der sog. "Äquidistanz" zwischen den Blöcken, also die Wahrung eines strikten Mittelkurses im Ost-West-Konflikt, während umgekehrt der Osten sich die Bewegung der Blockfreien als einen strategischen (oder "natürlichen") Verbündeten seiner Sache enger anzubinden versuchte. Beide Betrachtungsweisen der Blockfreien durch die Blockmächte stehen jedoch in einem fundamentalen Widerspruch zu den "Authentischen Prinzipien" der Blockfreiheit, denn in beiden Fällen wäre die politische Unabhängigkeit und eigenständige Handlungsfähigkeit der Bewegung gefährdet. ${ }^{15}$ Der Forderung nach "Äquidistanz" liegt deutlich eine Gleichsetzung des völkerrechtlichen, eher statisch-passiven Konzepts der "Neutralität" mit dem politischen, eher dynamisch-aktiven Konzept der "Blockfreiheit" zugrunde. ${ }^{16}$

13 Ebd., S. 51, 354; vgl. z. B. S. D. Munim, Non-Alignment and the Security Parameter, a.a.O.; S. B. Farajallah, The Security Challenges to the Non-aligned Movement, in: U. S. Bajpai (ed.), a.a.O.

14 Zur angeblichen "Krise" der Blockfreienbewegung siehe Ranko-Petković, Unhaltbare These über Krise der Blockfreiheit, in: Internationale Politik, H. 787 vom 20. Januar 1983.

$15 \mathrm{Vgl}$. hierzu Ranko Petković, The Authentic Principles of Non-alignment, in: Non-Alignment in the Eigthies, a.a.O.

16 Zum Verhältnis von Blockfreiheit und Neutralität siehe z. B. Jens Hacker, Neutralität, Neutralismus und Blockfreiheit, in: Aus Politik und Zeitgeschichte, Beilage zur Wochenzeitung das Parlament, B 18/83 vom 7. Mai 1983. 
Die genannte Kritik an den Blockfreien gründet sich auf die Annahme, daß (neben dem Anwachsen der Mitgliedschaft) vor allem neuartige sicherheitspolitische Probleme zu einer Erosion der Basiskriterien von Blockfreiheit und damit zugleich auch zu einer "Sinnkrise" des Konzepts von Blockfreiheit überhaupt geführt hätten. Nach den 1961 beschlossenen Kriterien galten und gelten folgende Staaten als "blockfrei":

"1. The country should have adopted an independent policy based on the coexistence of States with different political and social systems and non-alignment or should be showing a trend in favour of such a policy.

2. The country concerned should be consistently supporting the movements for National Independence.

3. The country should not be a member of a multilateral military alliance concluded in the context of Great Power conflicts.

4. If a country has a bilateral military agreement with a Great Power, or is a member of a regional defence pact, the agreement or pact should not be one deliberately concluded in the context of Great Power conflicts.

5. If it has conceded military bases to a Foreign Power, the concession should not have been made in the context of Great Power conflicts. $1{ }^{17}$

Diese fünf Basiskriterien von Blockfreiheit, und insbesondere die drei letzteren, welche die sicherheitspolitische Kernsubstanz des Konzepts der Blockfreiheit ausmachen, stellten von Beginn an eine Kompromißformel dar; der Streit unter den Blockfreien betraf die Legitimität, das Ausmaß und die Art einer militärischen Zusammenarbeit mit den Blockmächten. Mithin blieben die Kriterien in erheblichem Maße auslegungsfähig: wie vor allem ließ sich die zentrale Formel "nicht im Kontext von Großmacht-Konflikten" interpretieren? "Since non-alignment is not operationalized beyond non-participation in formal East-West military alliances, there is no unanimity on other uniform criteria to identify and classify a non-aligned actor. ${ }^{18}$

Im Laufe der Jahre ging eine Vielzahl blockfreier Länder im Zuge neuartiger sicherheitspolitischer Herausforderungen militärische Bindungen an die Block- und Supermächte ein, die kaum noch im Einklang mit dem Geist der Kriterien von 1961 standen. Uber Rüstungstransfers, Interventionen und "Stellvertreterkriege" hatte sich besonders in den 70ger Jahren der Ost-West-Konflikt zunehmend auf die Dritte Welt ausgedehnt, die sozusagen zu dessen "Süddimension" wurde: "instead of fighting exclusively with each other, the Super Powers are fighting, directly and indirectly, within and through the Third World. “19 Wichtige Ansatzpunkte für ein verstärktes Engagement der Supermächte boten die vermehrten "Bruderkriege" zwischen und die Konflikte in blockfreien Ländern. Um in dieser schwieriger gewordenen sicherheitspolitischen Situation ihre

17 Zitiert nach Odette Jankowitsch/Karl P. Sauvant (eds.), The Third World Without Superpowers: The Collected Documents of the Non-Aligned Countries, Vol. I, Dobbs Ferry-New York 1978, S. 38.

18 Zitiert nach Bahgat Korany, a.a.O., S. 159.

19 Zitiert nach S. D. Muni, Non-Alignment and the Security Parameter, a.a.O., S. 164. 
"nationalen Interessen" wahren zu können, ließen sich etliche blockfreie Staaten in Formen militärischer Kooperation mit den Block- und Supermächten ein, die zwar nicht immer im konventionellen Sinne einem "Militärpakt" entsprachen, also eher informeller Art waren, aber de facto einem solchen Abkommen oftmals doch gleichkamen und auch mehr oder weniger eindeutig im Zusammenhang mit Großmacht-Konflikten standen. Die neuen Formen militärischer Zusammenarbeit blockfreier Staaten mit blockgebundenen Staaten umfaßten u. a. die Bereitstellung von militärischen "Fazilitäten", die Abhaltung gemeinsamer Manöver, die Gewährung umfangreicher Militär- und Ausbildungshilfe sowie Unterstützung bei Logistik und militärischer Aufklärung bis hin zur Entsendung von Militärberatern oder gar Kampftruppen. Dies gilt z. B. für SaudiArabien, Kenia, Oman und Marokko im Hinblick auf die USA, für Kuba, Vietnam und Äthiopien im Hinblick auf die UdSSR, und für eine Anzahl frankophoner Staaten Afrikas im Hinblick auf Frankreich.

Angesichts dieser neuen sicherheitspolitischen Entwicklungen in der Dritten Welt hat die in den Basiskriterien von Blockfreiheit enthaltene Formel "nicht im Kontext von Großmacht-Konflikten" zunehmend "a ring of ambiguity about it" bzw. "it seems to have lost its meaning and relevance completely today ${ }^{20}{ }^{20}$ Dies vor allem als Folge der globalen Dimension des Ost-West-Konflikts, die bede utet, daß "any military agreement of a given non-aligned country with one of the great powers can, under certain circumstances, be linked to the East-West conflict which is expressly prohibited by the code of these criteria ". ${ }^{21}$ Ansätze zur Lösung der Problematik lägen vor allem in dem verstärkten Bemühen zur Eliminierung der Ursachen und friedlichen Regelung von Konflikten, die den Blockmächten Gelegenheit zur Einmischung geben, sowie ferner in einer intensiveren sicherheitspolitischen Zusammenarbeit der Blockfreien untereinander, die allerdings in deutlicher Distanz zu den Machtblöcken betrieben werden müßte. Von derartigen Ansätzen ist die Bewegung der Blockfreien jedoch noch weit entfernt; bislang begnügte sie sich noch immer mit der Bestätigung und dem Aufruf zur strikten Einhaltung der Kriterien von $1961 .^{22}$

20 Ebd., S. 167.

21 So Bojana Tadić, Survey of Discussions, in: Non-Alignment in the Eigthies, a.a.O., S. 433.

22 So die Erklärung der Staats- und Regierungschefs der blockfreien Staaten auf der 7. Gipfelkonferenz der Blockfreien im März 1983 in New Delhi (Schlußerklärung: Politischer Teil), zitiert nach Internationale Politik, H. 792 vom 5. April 1983: "Wiederum bestätigten die Staats- oder Regierungschefs die grundsätzliche Entscheidung der blockfreien Länder dafür, sich an keiner Aktion zu beteiligen, die die Konfrontation und Rivalität der Großmächte schüren oder die bestehenden Militärbündnisse und die mit ihnen verbundenen Abkommen stärken könnte, vor allem durch die Teilnahme an militärischen Abmachungen oder durch die Gewährung von Militärstützpunkten und Vergünstigungen für die militärische Präsenz der Großmächte im Zusammenhang mit ihren Konflikten. Erneut bestätigt wurden die Kriterien für die Teilnahme an der Bewegung, wie sie in Kairo formuliert und 1961 in Belgrad beschlossen wurden, und es wurde ihre strikte Einhaltung verlangt". Etwas deutlicher fourmulierte allerdings die neue Vorsitzende der Bewegung der Blockfreien, Indira Gandhi, in ihrer Eröffnungsansprache an die Konferenz: "Neue Bereiche wurden in strategische Gruppierungen, Militärblöcke und Bündnisse aufgenommen. Es werden neue Stützpunkte und Anlagen errichtet. Daher müssen wir konsequenter, rascher und schärfer antworten." Zitiert nach Internationale Politik, H. 792 vom 5. April 1983, S. 17. 


\subsection{Blockfreiheit und Abrüstung: Vorrangige Verantwortung der Block- und Super- mächte?}

Abrüstung gehört zu den "Gründungs- und Dauerthemen" der Bewegung der Blockfreien. Konzeptionell ist Abrüstung im Rahmen von Blockfreiheit in einen weiteren Zusammenhang eingefügt: in den Kampf gegen die Block- und Machtpolitik, in die Bemühungen um die Schaffung eines neuen Systems internationaler Beziehungen auf der Basis friedlicher Koexistenz sowie in den Kampf um die Errichtung einer Neuen Weltwirtschaftsordnung (= Ressourcenfreisetzung für wirtschaftliche Aufbauzwecke; Zusammenhang von Abrüstung und Entwicklung). Seit 1961 entfaltete sich die blockfreie Abrüstungsdiplomatie in den Vereinten Nationen und in dem Genfer AbrüstungsKomitee; doch wenngleich auch die Blockfreien in der Abrüstungsfrage kontinuierlich aktiv waren, so entwickelte sich aus dieser Arbeit doch kein "charakteristisches Aktivitätsprofil" . . ., das etwa mit den Blockfreien-Aktivitäten zur Neuordnung der weltwirtschaftlichen Beziehungen vergleichbar wäre».23 Gewisse Höhepunkte blockfreier Abrüstungsdiplomatie stellten die auf Initiative der Blockfreien hin einberufenen UN-Sonderkonferenzen über Abrüstung von 1978 und 1982 dar; auf diesen Konferenzen fand die blockfreie "Abrüstungsphilosophie" (vor allem Grundsatz der allgemeinen und vollständigen Abrüstung, besondere Verantwortung der Supermächte für die Abrüstung, vor allem für die atomare Abrüstung, Verbindung von Abrüstung und Ressourcenfreisetzung für friedliche Entwicklungszwecke, Vereinte Nationen als abrüstungspolitisches Forum mit universaler Beteiligung) erstmals volle internationale Anerkennung und Eingang in die Schlußdokumente. Doch kam es andererseits auf diesen Konferenzen weder zu verbindlichen und konkreten Abrüstungsverpflichtungen noch zu Zeitfestlegungen einzelner Abrüstungsschritte.

In der Sicht der Blockfreien kommt den Supermächten bei der Abrüstung, namentlich bei der Nuklearabrüstung, eine besondere weltpolitische Verantwortung zu. Durch den atomaren Rüstungswettlauf zwischen den Kontrahenten des Ost-West-Konflikts und die enorme Ressourcenverschwendung dieses Rüstungswettlaufs sehen sich die Blockfreien sicherheitspolitisch besonders bedroht und in ihrer wirtschaftlichen Entwicklung behindert. Abrüstungspolitische Priorität müssen daher die Bemühungen der Supermächte um die Verringerung ihrer Atomwaffenarsenale haben. Der Frage konventioneller Abrüstung - auch in ihren eigenen Reihen - widmen sich die Blockfreien dagegen nur mit geringerer Intensität. Zwar wurde die Parole von der allgemeinen und vollständigen Abrüstung nicht fallen gelassen, aber doch etwas in den Hintergrund gerückt: "Die eigene Abrüstung wird mit der allgemeinen Weltlage gerechtfertigt, wobei hervorgehoben wird, daß der Frieden durch das in den Arsenalen der Supermächte angehäufte Potential bedroht sei; die verhältnismäßig begrenzte Waffenmenge in den bedrohten Ländern stelle ohnehin keine Gefahr für den Weltfrieden dar. Diese Waffenvorräte

23 So Rüdiger Jütte, Blockfreiheit und Abrüstung, in: Internationale Entwicklung 1981/II, S. 7. 
könnten nur dann vernichtet werden, wenn die Großen zu erkennen gäben, daß sie das Abrüstungsproblem ihrerseits ernst nähmen. "124

Der Logik ihrer Forderung nach einer vorrangigen Abrüstung im Nuklearbereich folgend, haben die Blockfreien in ihrer Mehrheit eine grundsätzlich positive Haltung zur Nichtweiterverbreitung von Atomwaffen (Non-Proliferations-Vertrag/NPT) eingenommen. Doch gilt dies keineswegs für alle blockfreien Länder; eine Anzahl von ihnen (u. a. Algerien, Argentinien, Indien, Kuba, Pakistan) hat bis heute den NPT nicht unterzeichnet und/oder nicht ratifiziert. Einige dieser Länder, namentlich Indien, die sich aus verschiedenen Erwägungen heraus scheinbar auch die Nuklearoption im militärischen Sinne of fenhalten wollen, haben den NPT einer scharfen Kritik unterzogen. Der Vertrag sei "ungleich und diskriminierend", da die west- und osteuropäischen Bündnispartner der Supermächte als Quasi-Atomwaffenmächte angesehen werden könnten, und daher allein die Nicht-Nuklearstaaten (und atomaren "Schwellenländer") der Dritten Welt von den im Vertrag vorgesehenen scharfen Sicherheitskontrollen erfaßt würden. Insgesamt liege dem Vertrag die kaum zu akzeptierende Forderung der Nuklearstaaten zugrunde: "Wir können Euch nicht trauen, aber bitten Euch, uns zu trauen. ${ }^{25}$ Diese Ambivalenz werde vor allem auch daran deutlich, daß die Nuklearmächte selbst ihren Verpflichtungen aus dem Vertrag bislang nicht oder nur sehr unzureichend nachgekommen seien. Weder hätten sie sich bisher in konsequente Abrüstungsverhandlungen eingelassen noch in zureichendem Maße eine Weitergabe ziviler Nukleartechnologie ermöglicht. Auf diesem Hintergrund diene der NPT einzig und allein der Absicherung des derzeitigen Atom-Monopols der Nuklearmächte und der Verfestigung der überkommenen internationalen Machtstruktur. Ferner behinderten die Nuklearmächte durch ihre restriktiven Politiken bei der Weitergabe ziviler Nukleartechnologie die technologische und wirtschaftliche Entwicklung der Dritten Welt und trügen damit zur Vertiefung des Nord-Süd-Gegensatzes bei. ${ }^{26}$

Im Bereich der regionalen Abrüstung haben sich die Blockfreien (namentlich Sri Lanka und Indien) seit 1970 insbesondere für die Errichtung einer "Friedenszone Indischer Ozean" eingesetzt, die auf ihr Drängen hin Ende 1971 von der UN-Vollversammlung proklamiert wurde. Hauptziel dieser UN-Resolution war die Beseitigung sämtlicher ausländischer Stützpunkte und militärischer Einrichtungen sowie jeder Art von militärischer Großmacht-Präsenz im Rahmen der Supermacht-Rivalität in der Indik-Region. Eine sicherheitspolitische Relevanz des Konzepts der Friedenszone Indischer Ozean ist jedoch bis heute praktisch-politisch kaum gegeben. Die Ursachen hierfür scheinen in zwei mit diesem Konzept verbundenen schwierigen Grundproblemen zu liegen; zum einen in der Unklarheit des Konzepts selbst (Proliferation von Friedenszonen-Formeln;

24. Zitiert nach Leo Mates, Es begann in Belgrad. 20 Jahre Blockfreiheit, Percha am Starnberger See 1982, S. 300.

25 So M. Zuberi, Nuclear Safeguards and the Developing Countries: The Servitudes of Cicilian Nuclear Technology, in: International Studies, vol. 20, nos. 1-2, Januar-Juni 1981, S. 188.

$26 \mathrm{Vgl}$. allgemein zur Problematik T. T. Poulose, Nuclear Proliferation and the Third World, New Delhi 1982. 
Vorschläge u. a. von seiten der ASEAN, der Rotmeer-Anrainer, des Golf-Rates und Nepals; Schwierigkeiten auch bei der geographischen Abgrenzung der Indik-Region: Einbeziehung von Randmeeren?) und zum anderen in der realen Entwertung des Konzepts durch gegenläufige Aufrüstungsprozesse in der Region (Wandlung der "Zone des Friedens" in eine "Zone der Konflikte und Spannungen" im Zusammenhang mit der verschärften Supermacht-Rivalität im Zuge der sowjetischen Militärpräsenz in Äthiopien und der sog. "Doppelkrise" um Iran und Afghanistan).

Doch läßt sich das Scheitern der "Friedenszone" nicht allein auf extra-regionale Faktoren wie den verschärften Ost-West-Konflikt zurückführen, sondern auch auf intra-regionale Faktoren, also auf das sicherheitspolitische Verhalten der blockfreien Anrainerstaaten selbst. Intra-regionale Bedrohungsvorstellungen, Machtungleichgewichte und Konflikte haben ein gut Teil zur Aufrüstung und Großmacht-Einmischung im Bereich der Friedenszone beigetragen. Mancher blockfreie Anrainer, der für die Friedenszone eintritt, hat gleichzeitig die militärische Anlehnung an eine Supermacht betrieben und diese dadurch geradezu in die Friedenszone "hineingezogen". Durch mehr wirtschaftliche, politische und militärische Kooperation untereinander hätten die Blockfreien in der Region vielleicht eine Chance, dem Ausbruch von Konflikten und einer Einmischung der Großmächte vorzubeugen und diesen gegenüber ihr kollektives Verhandlungsgewicht zu erhöhen. ${ }^{27}$

Zusammenfassend läßt sich als wichtigstes Ergebnis blockfreier Abrüstungspolitik festhalten, daß den langjährigen "oligarchischen" internationalen Abrüstungsverhandlungen ein "demokratisches" Element zugefügt wurde. Allerdings handelte es sich bei dieser "Demokratisierung" internationaler Abrüstungspolitik eher um formale, prozedurale und konzeptionelle Zugeständnisse als um ein inhaltliches Entgegenkommen. In der Substanz haben die Blockfreien keines ihrer zentralen abrüstungspolitischen Ziele erreichen können: weder wurde der Rüstungswettlauf zwischen Ost und West gestoppt oder gar in einen Prozeß der Abrüstung verkehrt, noch wurden realpolitisch eine Friedenszone Indischer Ozean durchgesetzt und Ressourcen aus Abrüstungsmaßnahmen freigesetzt. Ohnehin gilt es heute zunehmend als unwahrscheinlich, daß im Sinne der Formel "Abrüstung und Entwicklung" "diese eher mechanische Vorstellung der Umschichtung der Mittel von Rüstung zu Entwicklung automatisch funktioniert". ${ }^{28}$ Schließlich nahm die Rüstung und Militarisierung (einschließlich der Waffenproduktion und des Waffenhandels) in der Dritten Welt selbst enorm zu. Nun läßt sich diese weitgehende Wirkungslosigkeit blockfreier Abrüstungspolitik sicherlich zu einem gut

27 Vgl. hierzu Dieter Braun, Arbeitspapier betr.: Indian Ocean: The "Peace Zone" Paradox and Chances for Areas of Peace, SWP-AP 2321, Juni 1982 und G. W. Shephard Jr., Demilitarization Proposals for the Indian Ocean, in: L. W. Bowman/Jan Clark (eds.), The Indian Ocean in Global Politics, Boulder, Col. 1981 sowie A. K. Damodaran, Indian Ocean: A Zone of Peace, in: Non-Aligned Issues. A Perspective, publ. by Ministry of External Affairs, Gov. of India, New Delhi, Januar 1981 und K. P. Misra, Indian Ocean: Peace Zone Concept, in: Indian \& Foreign Review vom 1. April 1982.

28 Vgl. hierzu Herbert Wulf, Einleitung: Abrüstung und Entwicklung, in: Ders. (Hrsg.), Aufrüstung und Unterentwicklung. Aus den Berichten der Vereinten Nationen, Reinbek bei Hamburg 1983, S. 20. 
Teil auf die relative Machtlosigkeit der Blockfreien gegenüber den hochgerüsteten Block- und Supermächten zurückführen. Doch müssen Gründe für diese ernüchternde Bilanz auch in dem rüstungspolitischen Verhalten der Blockfreien selbst gesucht werden. Mit Brock kann der blockfreie Grundsatz von der vorrangigen Verantwortung der Supermächte für die Abrüstung, insbesondere für die atomare Abrüstung, nur dann akzeptiert werden, wenn die Blockfreien gleichzeitig auch das Problem ihrer eigenen Aufrüstung nicht außer acht lassen. ${ }^{29}$ Der Ost-West-Konflikt kann - von Ausnahmen abgesehen - kaum ein Rechtfertigungsgrund für die Aufrüstung blockfreier Länder sein, da der Austrag dieses Konflikts in der Dritten Welt ja offenkundig oftmals erst durch Konflikte in und zwischen blockfreien Ländern ermöglicht wird. Andererseits soll nicht in Abrede gestellt werden, daß es durchaus legitime Gründe für ein angemessenes Maß an Rüstung in diesen Ländern geben kann. Dennoch kommt den Blockfreien eine erhebliche abrüstungspolitische Eigen- und Mitverantwortung zu. Wollen sie nicht mit ihrem Grundsatz der "allgemeinen und vollständigen Abrüstung" unglaubwürdig werden, müßten sie mehr als bisher zumindest die von ihnen "hausgemachten" Gründe für Aufrüstung auszuschalten versuchen. Konflikte in und zwischen ihren Ländern müßten friedlich beigelegt, die Ursachen für diese Konflikte nach Möglichkeit beseitigt werden, u. a. durch ausgewogene und sozial gerechte Entwicklungspolitiken; ferner müßten Rüstungsproduktion und -handel in und zwischen blockfreien Staaten begrenzt, alternative Militär-, Rüstungs- und Verteidigungspolitiken gefördert und Optionen auf einen militärischen Nuklear-Status aufgegeben werden, denn "die Politik der Bewegung wird auch schon durch die öffentliche und offizielle Proklamierung solcher Ambitionen und Pläne schwer genug belastet $"{ }^{30}$ Der Auffassung des Jugoslawen Mates zufolge sollte die Bewegung der Blockfreien auch "gegen einzelne extreme Fälle von Waffenhäufung, insbesondere aber gegen Ambitionen auf Kernwaffenbesitz, energischer und entschlossener" vorgehen. ${ }^{31}$

\section{Blockfreiheit und "Hausfrieden"}

\subsection{Der "Äußere Frieden«: Kriege und Friedliche Streitbeilegung zwischen Blockfreien}

Kriegerische Konflikte zwischen Blockfreien gab es von Beginn der Bewegung an, und insofern stellen diese "Bruderkriege" in der "Familie der Blockfreien" kein prinzipiell neues Phänomen dar. Doch seit etwa der zweiten Hälfte der 70ziger Jahre kam es zu Kriegen zwischen Mitgliedstaaten der Bewegung, die durch ihre Quantität und Qualität

29 Vgl. Lothar Brock, Disarmament und Non-alignment, in: Gandhi Marg. Special Issue on Disarmament and Human Survival, Nr. 38+39, New Delhi, Mai-Juni 1982.

30 So Leo Mates, Es begann in Belgrad, a.a.O., S. 302.

31 Ebd., S. 302. 
(u. a. Kriege zwischen Kampuchea/Vietnam; Äthiopien/Somalia; Uganda/Tansania; Iran/Irak; teilweise Züge von "Stellvertreterkriegen "; "Süddimension" des Ost-WestKonflikts) doch neuartige und schwerwiegende sicherheitspolitische Probleme für die Blockfreien aufwarfen. Zwar bedrohten diese Kriege nicht die Existenz der Bewegung als solche, aber sie beeinträchtigten doch in erheblichem Maße deren Einheit, Unabhängigkeit und Glaubwürdigkeit:

- Kriege zwischen Mitgliedstaaten gelten als "dysfunctional to the "cohesion" of the group as a whole and as adverse to their bargaining position "vis-á-vis" the blocs". ${ }^{32}$ Derartige Konflikte können die Mitgliedschaft spalten und die Energien der Bewegung über Gebühr beanspruchen (infolge des Krieges zwischen Iran/Irak mußte beispielsweise die 7. Gipfelkonferenz der Blockfreien kurzfristig von Bagdad 1982 auf New Delhi 1983 verlegt werden);

- Konflikte zwischen Blockfreien können durch "push- und pull-Faktoren" zu einer Einmischung der Super- und Blockmächte in Form von Rüstungstransfers oder offenen/versteckten Interventionen führen. Hierdurch würden sich diese Konflikte unnötig internationalisieren, das Risiko einer direkten Konfrontation zwischen den Supermächten erhöhen, dadurch zur Gefährdung des "Weltfriedens" beitragen und friedliche Konfliktlösungen verzögern und/oder erschweren. Durch Einmischung der Super- und Blockmächte aber werde das Hauptziel von Blockfreiheit, nämlich "to keep out of the relationship of major tension " ${ }^{33}$ gerade verfehlt. Infolge einer solchen Verstrickung in den Ost-West-Konflikt werde letztendlich nicht nur die Unabhängigkeit der unmittelbar beteiligten blockfreien Länder bedroht, sondern auch die von der Bewegung der Blockfreien insgesamt angestrebte unabhängige Rolle in der Weltpolitik;

- schließlich mindern solche "Bruderkriege" auch die Glaubwürdigkeit und das Ansehen der Bewegung als eine "moralische Kraft" in den internationalen Beziehungen. Da die Bewegung ja auf die Prinzipien der Friedlichen Koexistenz und der Friedlichen Streitbeilegung festgelegt ist, werden Kriege zwischen ihren Mitgliedern als ein von der selbst proklamierten Norm abweichendes Verhalten ausgelegt. So heißt es denn auch in einem von Jugoslawien zur Problematik kriegerischer Konflikte zwischen Blockfreien vorgelegten Papier: "It is necessary for the non-aligned countries to respect in their mutual relations the principles for whose universal application in international relations in general they have pledged themselves" ${ }^{34}$

Zur Bewältigung der durch Kriege zwischen Mitgliedstaaten aufgeworfenen Probleme hat die Bewegung der Blockfreien vor allem Anstrengungen auf der normativen Ebene (Bekräftigung des Prinzips friedlicher Streitbeilegung) und im konzeptionellen Bereich (Diskussion verschiedener operativer Ansätze zum Verfahren friedlicher Streitbeilegung) unternommen. Im konkreten Fall des Krieges zwischen Iran/Irak ist sie allerdings

32 So Bahgat Korany, Social Change ..., a.a.O., S. 370.

33 Ebd., S. 370.

34 Zitiert nach Delegation of Yugoslavia, Working Paper on Peaceful Settlement of Disputes between NonAligned Countries, NAC/FM/DEL/WP. 5,5 February 1981. 
erstmalig unmittelbar als kollektiver Vermittler (Komitee der Guten Dienste auf Ministerebene) um eine friedliche Streitbeilegung bemüht gewesen. Seit 1978 (Ministertreffen des Koordinierungsbüros in Havanna) haben sich verschiedene Konferenzen der Blockfreien mit der Frage befaßt, auf welche Weise und mit welchen Mitteln die Mitglieder der Bewegung - individuell oder kollektiv - möglichen Streitparteien bei der friedlichen Konfliktregelung helfen könnten. Als Diskussionsgrundlage dienten dabei ein von Sri Lanka eingebrachtes Arbeitspapier über eine Kommission zur friedlichen Beilegung von Grenzstreitigkeiten zwischen blockfreien Ländern, ein von Jugoslawien vorgelegtes Arbeitspapier über die Bereitstellung Guter Dienste durch die Blockfreien (als Ganzes, durch das Koordinierungsbüro, Ausschüsse, einzelne Länder oder durch Ad Hoc-Gruppen) sowie ein gemeinsam von Jugoslawien/Bangladesh/Irak erarbeiteter Resolutionsentwurf. ${ }^{35}$ All diese Konzepte zeichnen sich durch drei wesentliche Merkmale aus: 1) bevorzugen sie eher politische Streitbeilegungsverfahren (Gute Dienste, Vermittlung) gegenüber eher juristischen Verfahren (Vergleich, Schiedsspruch, Gerichtliche Regelung); 2) machen sie jegliches Bemühen der Blockfreien um Konfliktregelung von der Zustimmung der Streitparteien abhängig und 3) betrachten sie die Aktivitäten der Blockfreien weder als prioritär noch als exklusiv gegenüber Konfliktregelungsbemühungen von anderer Seite (z. B. von Regionalorganisationen wie der OAU). Mithin körınen die genannten Vorschläge in ihrer politischen Tragweite und in ihrem Institutionalisierungsgrad als recht begrenzt und bescheiden gelten, wobei allerdings die "Grenzkommission" Sri Lankas vergleichsweise stärker institutionalisiert und formalisiert erscheint als die von Jugoslawien favorisierten Ad-Hoc-Gruppen.

Doch bisher ist noch keine definitive Entscheidung über ein bestimmtes Verfahren friedlicher Streitbeilegung im Rahmen der Bewegung getroffen worden. Die 7. Gipfelkonferenz 1983 in New Delhi begnügte sich mit der Bekräftigung, "daß der Grundsatz der friedlichen Lösung von Streitigkeiten das Kernprinzip der Philosophie der friedlichen Koexistenz bleibt, die von den blockfreien Ländern vertreten wird “. ${ }^{36}$ Jaipal spricht in diesem Zusammenhang von einer "allgemeinen Allergie" der Blockfreien gegenüber einer wie auch immer gearteten Institutionalisierung der Konfliktregelung. ${ }^{37}$ Zweifellos

35 Ebd. sowie Sri Lanka Delegation, Working Paper on the Establishment of a Commission of Border Disputes within the Non-Aligned Moevement, NAC/FM/DEL/WP. 6,5 February 1981; Resolution on the Peaceful Settlement of Disputes among Non-Aligned Countries, submitted by Bangladesh, Iraq and Yugoslavia, abgedruckt in Peter Willetts, The Non-Aligned in Havana, London 1981, S. 228-231.

$36 \mathrm{Zu}$ diesem Grundsatz führte die Konferenz weiter aus: "Sämtliche Streitigkeiten sind ausschließlich mit friedlichen Mitteln, in völliger Ubereinstimmung mit den Zwecken, Prinzipien und Bestimmungen der Charta der Vereinten Nationen, den Prinzipien und Zielen der blockfreien Bewegung sowie im Rahmen und entsprechend den Grundsätzen regionaler Organisationen wie der Organisation für die Einheit Afrikas (OAU), ohne Einmischung und Anwendung von Druck, Gewaltandrohung oder -anwendung zu regeln. Streitigkeiten zwischen Nachbarländern müssen durch friedliche Mittel, auf dem Wege direkter Verhandlungen, durch Vermittlung oder gute Dienste seitens Interessierter oder durch andere in der Charta der Vereinten Nationen vorgesehenen Maßnahme beigelegt werden, und zwar, ohne zu Druck, Drohungen oder Gewaltanwendung, ausländischer Intervention oder Einmischung Zuflucht zu nehmen. "Zitiert nach Schlußerklärung (Politischer Teil), abgedruckt in Internationale Politik, H. 792 vom 5. April 1983.

37 So Rikhi Jaipal, Non-Alignment. Origins, Growth and Potential for World Peace, New Delhi usw. 1983, S. 146. 
handelt es sich bei der Konfliktregulierung, da sie eng mit Sicherheits- und Souveränitätsfragen verknüpft ist, um ein sensibles und brisantes Thema. Allgemein haben die Blockfreien bislang eher "weiche" Organisationsstrukturen (z. B. Rotation des Vorsitzes der Bewegung, Konsensbeschlüsse, Verzicht auf Statuten/Charten und auf ständige Sekretariate/Stäbe) bevorzugt. Komatina zufolge gibt es daher unter den Blockfreien "Zurückhaltung gegenüber jedem Schritt, der zur Annahme neuer Rechtsnormen und ... zu Verfahren führen würde, die auch nur eine Ankündigung obligatorischer Bestimmungen enthalten würden “. ${ }^{38}$ Etliche blockfreie Länder scheinen auch der Auffassung zu sein, daß die Regelung von Konflikten zwischen Mitgliedern am besten im Rahmen von Regionalorganisationen oder der Vereinten Nationen zu betreiben sei. Auf diesem Hintergrund könnten daher am ehesten noch die ausdrücklich informellen, flexiblen und pragmatisch auf den einzelnen Konfliktfall zugeschnittenen Ad Hoc-Gruppen des jugoslawischen Vorschlags eine Chance haben, von einer größeren Zahl blockfreier Länder gewürdigt zu werden.

Im Kontext der Bemühungen der Blockfreien um friedliche Streitbeilegung kommt dem Krieg zwischen Iran/Irak und den diesbezüglichen Vermittlungsbemühungen der Bewegung die exemplarische Bedeutung eines "Testfalls" zu. Die (bislang allerdings erfolglos gebliebene) Vermittlung der Blockfreien (durch das Präsidiumsland Kuba, durch ein Minister-Komitee der Guten Dienste, durch verschiedene Mitglieder während der 7. Gipfelkonferenz) in diesem Krieg war deren erster kollektiver Versuch, das Prinzip friedlicher Streitbeilegung zwischen Mitgliedern operativ umzusetzen. ${ }^{39}$ Das Scheitern dieses Versuchs sollte jedoch kein voreiliges Verdikt über die grundsätzliche Fähigkeit der Blockfreien zur Konfliktregelung nach sich ziehen. Denn zum einen sind ja auch parallel laufende Vermittlungsbemühungen anderer Akteure (u. a. Islamische Konferenz, UN-Mission Palmes) gleichfalls gescheitert; zum anderen unterlagen die Blockfreien den üblichen Restriktionen, denen ein jeder Vermittler in einem Konflikt ausgesetzt ist. Nach aller Erfahrung und theoretischer Erkenntnis ist nämlich der Erfolg einer Vermittlungsaktion von bestimmten Qualitäten des Vermittlers (u. a. strikte Unparteilichkeit) sowie von bestimmten Konfliktbedingungen (u. a. relative Machtsymmetrie, keine vitalen Sicherheits- und Territorialfragen) abhängig. ${ }^{40}$

Auf der normativen Ebene scheint das Kernproblem für die Bewegung der Blockfreien darin zu bestehen, wie denn ihre Mitglieder präventiv dazu angehalten werden könnten, gar nicht erst gegen die selbst gesetzten Prinzipien zu verstoßen. Um eine striktere Einhaltung der blockfreien Norm friedlicher Streitbeilegung zu gewährleisten, haben Wissenschaftler den Vorschlag unterbreitet, bei Mißachtung blockfreier Prinzipien intensiver als bisher die Frage von Sanktionen (bis hin zum Ausschluß!) gegen Mitglie-

38 Miljan Komatina, Die Blockfreiheit: Unterschiede und Streitigkeiten, in: Internationale Politik, H. 768 vom 5. April 1982, S. 9.

39 Ebd., S. 9 und Stanko Nick, Friedliche Beilegung der Streitigkeiten zwischen blockfreien Ländern, in: Internationale Politik, H. 743 vom 20. März 1981, S. 18.

40 Vgl. hierzu allgemein Volker Matthies, Der Grenzkonflikt Somalias mit Äthiopien und Kenya, Hamburg 1977, S. $362 \mathrm{ff}$. 
der zu erörtern (Petrić). ${ }^{41}$ Andere haben die Idee propagiert, durch eine feierliche Bekräftigung des Grundsatzes friedlicher Streitbeilegung durch jedes einzelne blockfreie Land (Vukadinović) ${ }^{42}$ oder durch die Verabschiedung eines besonderen blockfreien "Verhaltens-Kodex" (Degan) ${ }^{43}$ die normative Hemmschwelle für einen gewaltsamen Konfliktaustrag zu erhöhen. Im Interesse einer verbesserten Handlungsfähigkeit der Bewegung hat Mates gar die folgenschwere Frage aufgeworfen, ob nicht eine Abkehr von dem gängigen Konsensprinzip eine raschere und eindeutigere Klärung von Konfliktfällen durch Mehrheitsbeschluß ermöglichen könnte. ${ }^{44}$ Zur präventiven Konfliktverhütung hat ferner Willetts vorgeschlagen, von seiten der Blockfreien den internationalen territorialen Status Quo zu bekräftigen und eine Registrierung von Grenzen und Grenzverträgen durch die Vereinten Nationen vornehmen zu lassen. ${ }^{45}$

Weitgehend unbefriedigend bleibt die Analyse der Ursachen der "Bruderkriege" durch die Blockfreien. Zwar wird in offiziellen Arbeitspapieren und in Forschungsarbeiten zur Thematik durchaus die Komplexität des Ursachenproblems angesprochen (u. a. territoriale Probleme, wirtschaftliche Rivalitäten, ideologische Streitigkeiten, raumfremde Einmischungspolitik als Ursachen), doch bleiben vor allem innergesellschaftliche Ursachen von zwischenstaatlichen Kriegen (wie z. B. die Gewinn-Interessen bestimmter sozialer Gruppen oder Klassen, die Externalisierung interner Spannungen) weithin ausgespart. Insbesondere bei dem häufigen Hinweis auf Grenz- und Territorialprobleme ist unklar, ob es sich denn hierbei um die wirklichen, tieferliegenden "Ursachen" oder aber nur um die "Anlässe" oder "Rechtfertigungsgründe" eines Konflikts handelt. ${ }^{46}$ Dies ist insofern von Bedeutung, als die Art und Weise der Regulierung eines Konflikts nicht zuletzt auch von der Beschaffenheit seiner Ursachen abhängig ist. Darüber hinaus ist eine dauerhafte Regelung eines Konfliks ja nicht allein durch seine "friedliche Beilegung" (im Sinne eines Verfahrens) zu erreichen, sondern letztlich nur im Zuge einer Minderung oder gar Ausschaltung seiner Ursachen.

\subsection{Der "Innere Frieden": Die "Sicherheit des Staates" und die "Sicherheit des Volkes"}

Eine kollektive öffentliche Debatte über Fragen der inneren Ordnung und Sicherheit blockfreier Länder hat es im Rahmen der Bewegung bisher nicht gegeben. Themen der inneren Ordnung und Sicherheit gelten bei den Blockfreien - wie überall in der Staaten-

41 Vgl. Ernest Petrić, Peaceful Settlement of Disputes among Non-aligned States, Paper at the IX. IPSA Congress, Rio de Janeiro, August 1982.

$42 \mathrm{Vgl}$. Radovan Vukadinović, Conflicts between Non-aligned Countries and Possibilities for Resolving Them, in: Non-alignment in the Eigthies, a.a.O.

$43 \mathrm{Vgl}$. V. D. Degan, Resolution of Disputes and Conflicts within the Non-aligned Movement, in: Nonalignment in the Eigthies, a.a.O.

$44 \mathrm{Vgl}$. Leo Mates, The Movement is Facing Discord and Trial, in: U.S. Bajpai (ed.), Non-Alignment, a.a.O.

$45 \mathrm{Vgl}$. Peter Willetts, The Future of the Institutions of the Non-aligned Movement, in: Nonalignment in the Eighties, a.a.O.

46 Vgl. hierzu allgemein Volker Matthies, Der Grenzkonflikt Somalias ..., a.a.O., S. $10 \mathrm{ff}$. 
welt - als ausgesprochene "Tabu"-Themen, die der ausschließlichen Souveränität des einzelnen Landes unterliegen. Doch gibt es hierfür auch eine blockfreienspezifische Erklärung. Als eine Gruppierung von Staaten sehr unterschiedlicher gesellschaftlicher und politischer Systeme hat sich die Bewegung der Blockfreien dennoch im Sinne einer "Einheitsfront-Strategie" der Verfolgung fundamentaler gemeinsämer Interesssen und Ziele ihrer Mitglieder verschrieben. Dieser strategisch-taktische Konsens schiene bedroht, wenn die Gruppenmitglieder sich untereinander über ihre inneren Angelegenheiten auseinandersetzen würden. Nur bei Tolerierung der politisch-sozialen Vielfalt ihrer Mitgliedschaft scheint die Bewegung ihre politische Einheit und internationale Handlungsfähigkeit bewahren zu können. In aller Deutlichkeit hat diesen Tatbestand mehrfach der tanzanische Staatspräsident Nyerere hervorgehoben, u. a. auf der 6. Gipfelkonf erenz der Blockfreien 1979 in Havanna, wo er gegenüber Castro deutlich machte, daß die Bewegung der Blockfreien zwar eine "progressive Bewegung" sei, jedoch "keine Bewegung progressiver Staaten" ${ }^{47}$

Auf der normativen Ebene schützen sich die Blockfreien gegen eine Ent-Tabuisierung der Probleme ihrer inneren Ordnung und Sicherheit mit den Prinzipien der Friedlichen Koexistenz zwischen Staaten unterschiedlicher Gesellschaftsordnung sowie der NichtIntervention und Nicht-Einmischung in die inneren Angelegenheiten von Staaten. ${ }^{48}$ Doch in der politisch-gesellschaftlichen Wirklichkeit blockfreier Staaten bestehen vielfältige Probleme der inneren Ordnung und Sicherheit. Etliche Länder wurden und werden von Bürgerkriegen heimgesucht, andere wurden von Revolutionen erschüttert oder sehen sich Guerilla- und Sezessionsbewegungen konfrontiert. Grundsätzlich könnte man bei Fragen der inneren Sicherheit zwischen der "Sicherheit des Staates" (= die Sicherheit der herrschenden Machtgruppen und des Staatsapparates) und der "Sicherheit des Volkes" (= die Sicherheit der Bevölkerung insgesamt, von Teilgruppen der Bevölkerung wie z. B. Minderheiten und von Einzelpersonen) unterscheiden. ${ }^{49}$ Während der "Staat" sich "von unten" her bedroht fühlt, z. B. durch Putschisten, Revolutionäre, Guerillas und Sezessionisten, sieht sich das "Volk" oftmals "von oben" her in seiner Sicherheit gefährdet, sei es durch allgemeine politische Unterdrückung und Verfolgung, sei es durch Diskriminierung, Vertreibung oder Vernachlässigung von Minderheiten, oder sei es durch die Mißachtung/Verletzung individueller Menschenrechte.

Zu Fragen des Minderheitenschutzes und der Menschenrechte gab es nur hin und wieder kurze Passagen in den Dokumenten der Blockfreien (so auf der 1. Gipfelkonferenz in Belgrad 1961, dann erst wieder auf der 6. Gipfelkonferenz in Havanna 1979; auf der Außenministerkonferenz in New Delhi 1981 und auf der 7. Gipfelkonferenz in New

47 Vgl. hierzu Volker Matthies, Die Sechste Gipfelkonferenz der Blockfreien in Havanna, in: Gegenwartskunde 1980/2.

48 Vgl. hierzu Ranko Petković, Non-intervention and Non-interference within the System of Collective Security and Non-aligned Policy, in: K. P. Misra/K. R. Narayanan (eds.), Non-Alignment in Contemporary International Relations, New Delhi 1982.

49 Begrifflichkeit nach Yoshikazu Sakamoto, Key Issues of Peace Research, in: International Peace Research Newsletter $3 / 81$. 
Delhi 1983). Trotz zahlreicher Regimewechsel und Umwälzungen der inneren Ordnung in etlichen blockfreien Ländern ist über die Jahre hinweg eine erstaunliche Konstanz der Mitgliedschaft in der Bewegung der Blockfreien festzustellen. $\mathrm{Zu}$ dem de facto-Ausschluß eines blockfreien Landes infolge seiner inneren Ordnung kam es bislang einzig im Falle Chiles, das nach dem gewaltsamen Sturz des Allende-Regimes durch die Militärs 1973 keine Einladung mehr zu den Konferenzen der Blockfreien erhielt.Eine Anzahl von Ländern wurden von den Blockfreien aufgrund ihrer inneren Situation auch positiv hervorgehoben oder mit einer neuen gesellschaftlichen Qualität als Mitglieder in die Bewegung aufgenommen, so z. B. auf dem Gipfel 1979 Panama wegen seiner neuen Revolutionsregierung und Nicaragua und der Iran nach ihren Siegen über die Shah- und Somoza-Diktaturen.

Hinweise auf die Haltung der Blockfreien zu Fragen der inneren Ordnung ihrer Mitglieder lassen sich auch den ökonomischen und entwicklungspolitischen Teilen ihrer Dokumente entnehmen. Hierbei geht es im Kern um die Konzepte der "Neuen Weltwirtschaftsordnung" und der "Kollektiven Self-Reliance". Diesen Konzepten läßt sich wenngleich auch oft nur vage und implizit - entnehmen, daß die Blockfreien letztendlich Gesellschaftsordnungen anstreben, die in der Lage sind, gerechte Sozial- und Einkommensverhältnisse sowie Massenbeschäftigung zu ermöglichen, und die zu einer Einbeziehung der Volksmassen in den Entwicklungsprozeß sowie zu einer Befriedigung menschlicher Grundbedürfnisse führen.

Auf dem Hintergrund der Tabuisierung der inneren Ordnung und Sicherheit gilt die Blockfreien-Bewegung manchen Kritikern als eine Art von "Heiliger Allianz" zur Absicherung des politisch-gesellschaftlichen Status Quo in ihren Mitgliedsländern. Die Machtgruppen der blockfreien Länder stellten die "Sicherheit des Staates" weit über die "Sicherheit des Volkes" und bedienten sich des Konzepts der Blockfreiheit oftmals nur als einer Legitimationsstrategie für ihre Dominanzposition. In Wirklichkeit seien die herrschenden Eliten blockfreier Länder nicht selten selbst die "worst enemies of the political unity, territorial integrity and wellbeing of the peoples of their countries".$^{50}$ Nach außen hin, im internationalen Bereich, forderten diese Eliten die Respektierung und Einlösung so hehrer Grundsätze und Ziele wie "Demokratisierung" das "Selbstbestimmungsrecht der Völker", "Emanzipation", die Beendigung von "Ausbeutung" und "Diskriminierung" sowie die Schaffung aller möglichen "Neuen Ordnungen" (Neue Weltwirtschaftsordnung, Neue Internationale Informationsordnung, Neue Weltmeeresordnung etc.), während sie im innergesellschaftlichen Bereich kaum auch nur etwas davon selbst respektierten oder einlösten. Brock spricht in diesem Zusammenhang ("Self-Reliance") von einer tiefen Kluft zwischen der "high politics" und der "low politics" von Blockfreiheit. ${ }^{51}$ Andere Forscher sehen durch dieses Verhalten der Eliten

50 So K. P. Misra in: Nonalignment. Tasks and Issues, issued under the auspices of Jawaharlal Nehru University, New Delhi, 1983, S. 8 f.

51 Lothar Brock, Human Needs, Self-Reliance and East-West Competition in the Third World: The Challenge of Nonalignment Today. Paper presented to the 9th General Conference of the International Peace Research Association, June 21-26, 1981, Orillia/Ontario, Canada. 
vor allem auch die Glaubwürdigkeit der Prinzipien und Ziele und des Anspruchs auf eine besondere moralische Kraft von Blockfreiheit gefährdet. Sie halten dafür, daß letztenendes nur eine konsequente Menschenrechtspolitik und ein Mehr an Demokratie der Blockfreiheit langfristig wirkliche Substanz und Uberzeugungskraft geben könnten. Zwar handele es sich bei den Fragen der inneren Ordnung und Sicherheit um eine "essentially domestic responsibility ",52 aber dennoch sollte die Bewegung der Blockfreien insgesamt doch zumindest zu einer stärkeren Sensibilisierung der Regierungen und der Offentlichkeit in blockfreien Ländern für mehr Menschenrechte und soziale Gerechtigkeit beizutragen versuchen. Auf diese Weise könnte vielleicht ein indirekter politischer Druck auf eine Wandlung der Verhältnisse erzeugt werden. Nur eine ausgewogene und sozial gerechte politische Ordnung und sozio-ökonomische Entwicklung könne "echte Stabilität" gewährleisten, zu einer annähernden Kongruenz der "Sicherheit des Staates" mit der "Sicherheit des Volkes" führen und damit letztlich den "Inneren Frieden" eines Landes ermöglichen: ". . the principal battle of each of the nonaligned is at home. Unless states are just and viable, unless they check ... the propensity toward dictatorship, they will collapse and succumb to new alignments... . No summit conference can resolve basic questions of political order and legitimacy. ${ }^{53}$

\section{4. "Friedens-Funktion" der Blockf reien im Internationalen System?}

Die These von einer "Friedens-Funktion" der Blockfreien im internationlen System muß als überzogen eingeschränkt werden. Die Blockfreien vermochten weder eine wachsende Verstrickung von Teilen ihrer Mitgliedschaft in den Ost-West-Konflikt und in die Blockpolitik verhindern, noch waren sie in der Lage, Kriege in den eigenen Reihen zu unterbinden oder friedlich zu lösen, effektive Zonen des Friedens zu errichten oder auf substantielle Weise zur Abrüstung beizutragen. Den neuartigen sicherheitspolitischen Herausforderungen seit Mitte der 70ziger Jahre begegneten sie zu spät, zu wenig originell und mit zu wenig Nachdruck. Konzeptionell schienen die Blockfreien zu stark in westlichen Sicherheits- und Verteidigungsdoktrinen befangen zu sein, denen sie stärker alternative sicherheitspolitische Ansätze entgegenstellen müßten: "This could be done by the non-aligned countries only by developing their own strategic thought. The ideas and concepts developed and promoted by the military-industrial-academic-mediacomplex of the West should be countered by the non-aligned countries. $1{ }^{54}$ Auch realpolitisch blieben die Blockfreien in erheblichem Maße in das Geflecht herkömmlicher Allianz- und Sicherheitspolitik verwoben, wenngleich auch meist auf eine eher indirekte, subtile und informelle Weise, die sie aber dennoch nicht die eigentlich

52 So S. B. Farajallah, The Security Challenges ..., a.a.O., S. 88.

53 Zitiert nach Fouad Ajami, The Fate of Non-Alignment, in: Foreign Affairs, no. 2, Winter 1980/81, S. 383.

54 Zitiert nach Summary of Discussions, in: K. P. Misra/K. R. Narayanan (eds.), a.a.O., S. 276. 
geforderte sicherheitspolitische Distanz zu der Blockpolitik und den Großmachtkonflikten einhalten ließ. Angesichts dieser Situation hält es Muni für eine der wichtigsten Aufgaben der Bewegung "to rebuild its security-strategic consensus which can define and execute the extent of proximity or distance that a non-aligned country should, and would, maintain in relation to a Superpower militarily ".55 Wie schwer eine solche Aufgabe allerdings zu lösen ist, verdeutlichte der tanzanische Staatspräsident Nyerere: "It is clear that we all need to try to maintain, or to reestablish as quickly as possible, our independence of any military connection with a super power. And if any of us fail, it is legitimate for the Non-Aligned Movement to question how and why it happened; not because there could be any prospect of excluding a member, but because we are all affected by the resultant reduction in our Movement's credibility. It is possible also that the prospect of our questioning may help a nation at risk to withstand the establishments or even the threats, of a super power.

Blanket condemnation of military links by Non-Aligned nations, however, is not only unjust and unrealistic. It can be used very easily as a device to weaken, divide, or undermine the Movement which has grown up. It is also a dangerously blinkered view of world realities. ${ }^{56}$

Andererseits kann auch weiterhin nicht geleugnet werden, daß zumindest in einem elementaren Sinne "the spirit of Non-Alignment does imply a restraint on the extent and intensity of the links between the Non-Aligned powers and the Super-Powers".57 Historisch gesehen bestand der wohl wichtigste Beitrag der Blockfreien zur internationalen Sicherheit in der Schaffung eines "Unabhängigkeitsgürtels" (Komatina), ${ }^{58} \mathrm{der}$, so "locker" er auch immer "geschnürt" gewesen sein mag, offenbar dennoch ein gewisses $\mathrm{Ma}$ an "struktureller Flexibilität" (Korany) ${ }^{59}$ in das internationale System einbrachte und damit zur Auflockerung allzu rigider Blockstrukturen beitrug und eine Ausdehnung der Blöcke über die ganze Welt verhinderte. Hier läge auch weiterhin eine wichtige Aufgabe für die Blockfreien. In dem neuerlich verschärften Ost-West-Konflikt sollten sie die Funktion wahrnehmen, das mit diesem Konflikt auf seiten der Supermächte verknüpfte "manichäische Denken" (in Gut und Böse) und mechanische "Null-Summen-Spiel“-Denken (in der globalen Auseinandersetzung zwischen den Blöcken gelten die "Gewinne" der einen Seite automatisch als die "Verluste" der anderen Seite) zu desavouieren und ad absurdum zu führen.

Allerdings war und ist die Festigkeit der blockfreien "Unabhängigkeitsgürtels" durch die oftmals zu geringe sicherheitspolitische Distanz vieler blockfreier Staaten zu den

55 S. D. Muni, Non-Alignment and the Security Parameter, a.a.O., S. 172.

56 So Nyerere in Daily News vom 7. März 1983.

57 So R. V. R. Chandrasekhara Rao, The Security Interests of the Non-Aligned, in: A. Perspective, in: Current Problems of the Non-Aligned Movement, Bandaranaike Centre for International Studies, Colombo/Sri Lanka, Januar 1981, S. 2.

58 So Miljan Komatina, Blockfreiheit und Sicherheit, a.a.O., S. 15.

59 So Bahgat Korany, Social Change . ., a.a.O., S. 364. 
Machtblöcken fortwährend gefährdet. Dabei reflektierte die Fragwürdigkeit der Kriterien von Blockfreiheit angesichts neuartiger sicherheitspolitischer Herausforderungen nur das fundamentale sicherheitspolitische Dilemma der Blockfreien, "of on one hand rejecting the powers but on the other hand having to depend on them economically and militarily" ${ }^{60}$ Diesem Dilemma vermöchten die Blockfreien vielleicht dadurch zu entgehen, daß sie sich in Úbernahme vermehrter sicherheitspolitischer Eigenverantwortung verstärkt den von ihnen "hausgemachten" Problemen zuwenden würden, deren NichtLösung sie bisher immer wieder zur Anlehnung an die Block- und Supermächte nötigt. In diesem Sinne könnte ein wichtiger Beitrag der Blockfreien zum "Weltfrieden" denn auch in der Herstellung und Absicherung ihres "Hausfriedens" liegen. Durch eine konsequentere Betreibung von Abrüstungsmaßnahmen in den eigenen Reihen (Begrenzung und Kontrolle konventioneller Rüstung, eindeutige Aufgabe der militärischen Nuklearoption) sowie durch eine präventive Verhütung oder friedliche Beilegung von inner- und zwischenstaatlichen Konflikten könnten die Blockfreien etliche Gefahrenquellen nicht nur für ihre eigene Sicherheit vermindern oder gar beseitigen, sondern auch für die internationale Staatengemeinschaft. Die wichtigsten Mittel und Methoden zur Erreichung dieser Ziele lägen in einer intensivierten und verbesserten Süd-Süd-Kooperation. Im Rahmen einer vielfältigen politischen, militärischen und wirtschaftlichen Zusammenarbeit hätten die Blockfreien Kooperationsstrukturen sowie gegenseitiges Vertrauen aufzubauen und untereinander friedfertiges Verhalten einzuüben. Auf diese Weise sollten auch "Friedenszonen" nicht nur postuliert und propagiert, sondern durch "dependable structures for defence and mutual security" konkret ausgefüllt werden. ${ }^{61}$ Zumindest auf der normativen Ebene hat die Bewegung der Blockfreien von ihren Absichten, Zielen und Prinzipien her immerhin das klassische "Macht-Paradigma" in den internationalen Beziehungen radikal infrage gestellt. Als Alternative bietet sie ein "Kommunikations-Paradigma" und entwirft sie ein "futuristisches Modell" internationaler Beziehungen, in dem "Macht" durch "Kommunikation" ersetzt wird und Kooperation statt Konfrontation vorherrscht. ${ }^{62}$ Die Bewegung propagiert damit Blockfreiheit als einen neuen "Verhaltens-Standard" in der internationalen Politik, der die Verkehrsformen der klassischen Staatenwelt (Allianz-, Hegemonial- und Machtpolitik zur Sicherung von Nationalinteressen) langfristig ablösen und ersetzen soll. Zur konkreten Annäherung an solche Visionen haben Wissenschaftler den Blockfreien vorgeschlagen, die bereits in ihren Reihen vorhandenen nicht-staatlichen Elemente (Befreiungsbewegungen und internationale Organisationen als Teil der Mitgliedschaft) zu stärken, das Konzept der staatlichen Souveränität zu relativieren und schließlich ein "transnationales

60 So Shaukat Hassan, Non-alignment: The Need for Self-scrutiny, in: Nonalignment in the Eigthies, a.a.O.

61 So S. D. Muni, Non-Alignment and the Security Parameter, a.a.O., S. $169 \mathrm{ff}$.

62 Vgl. hierzu J. W. Burton, International Relations. A General Theory, London usw. 1965, bes. Teil V.; Jayantanuja Bandyophadhyaya, The Non-Aligned Movement and International Relations, in: India Quarterly, vol. 33, 1977, S. 137-164; Peter Willetts, The Non-Aligned in Havana, London 1981, S. 41 ff.; K. P. Misra, The Conceptual Profile of Non-Alignment, in: K. P. Misra/K. R. Narayanan (eds.), a.a.O., S. 203 ff. 
System" anzustreben, das den Menschen mit seinen Grundbedürfnissen in den Mittelpunkt aller blockfreier Politik rückt.63

Doch auf Sicht wird die Bewegung der Blockfreien ein Verband von primär staatlichen Akteuren bleiben, die Teil des überkommenen Systems von "Nationalstaaten" sind, und deren Herrschaftsgruppen oftmals im Namen eines vorgeblichen "Nationalinteresses" nur ihre eigenen Interessen zu wahren trachten. Dies schließt allerdings nicht aus, daß die Blockfreien auch weiterhin bemüht sein werden, vor allem auf der normativen Ebene und im Rahmen der Vereinten Nationen (Weiterentwicklung des Völkerrechts, Stärkung des Gedankens universaler kollektiver Sicherheit, konzeptionelle Verknüpfung von militärischer und wirtschaftlicher Sicherheit, von Abrüstung und Entwicklung) zumindest zu einer teilweisen Verbesserung oder gar Neukonstruktion dieses Systems beizutragen. Ob jedoch die "Utopie" eines qualitativ neuen Systems internationaler Beziehungen wirklich einmal "konkret" werden könnte, wird nicht zuletzt auch von. dem Verhalten der Blockfreien selbst abhängen.

63 Vgl. hierzu Hanspeter Neuhold, Non-alignment: Some Basic Dilemmas and Difficult Choices, und Peter Willetts, The Future of the Institutions of the Nonaligned Movement, beide in: Nonalignment in the Eigthies, a.a.O. 


\title{
ABSTRACTS
}

\section{Rural Women's Legal Status in Ethiopia}

\author{
By Daniel Haile
}

The paper briefly discusses the legal status of rural women within the family (intra-familial) and their status outside it (extra-familial). Eventhough due to time constraint case studies could not be conducted on the basis of available material and earlier studies it has been found that the status of women within the family in post-revolution Ethiopia has been enhanced immensely, However, their status outside the family or in the community does not show commensurate progress. One factor that has hindered such progress has been their non participation in the key decision making organs of the rural community: the Peasant Association. Eventhough the law did not make any discrimination on the basis of sex narrow interpretation had resulted in granting membership rights to household heads thereby excluding the majority of women. Subsequent laws have tried to rectify this by specifically providing that both male and female have the right to become members of these associations and the author is confident that the state of affairs will soon be changed for the better.

However, despite the radical changes in the status of women, family relationships are still governed by the Civil Code of 1960. In light of this the author recommends that these laws be revised to be in tune with the changed reality. Furthermore as the role of women's associations in organizing women, educating them and implementing laws and policies is immense and every opportunity must be accorded to REWA (Revolutionary Ethiopia Women's Association) to participate in policy formulation and implementations.

\section{The Movement of the Non-Aligned - "Greatest Peace Movement in History»?}

\section{By Volker Matthies}

Non-Alignment has important national defence and security aspects, but the NonAligned States do not desire to, and cannot, constitute another military bloc. The NonAligned Movement is the champion of a universal international security system under the auspices of the United Nations. According to Nehru one had to create in Afro-Asia an "area of peace", whose "mobilization of the moral violence of people" against "situations of strength" and military alliances would introduce an element of systemic flexibili- 
ty, act as a bridge-builder and a communication channel, and mediate to reduce the level of conflict. When the Non-Aligned States tried to keep out of the East-West tensions they were faced with the dilemma of on one hand rejecting the major powers but on the other hand having to depend on them economically and militarily. In recent years, due to new security challenges (New Cold War, wars among Non-Aligned Countries), NonAlignment in general and its efficacy as a policy reflecting the underlying security concerns in particular, have come under severe strain.

\section{The dilemma facing industrialized countries in respect to the refugee problem in Asia}

\section{By Gudrun Lachenmann}

The world refugee problem has been treated so far mainly as one of totalitarian régimes violating human rights (as far as its origins are concerned) and as one of international law and humanitarian aid (as far as its settlement is concerned). As a concequence actions taken can only attack its symptoms without touching underlying structural causes which can be analysed as an expression of the North-South-conflict.

This situation can be explained by the interests of the industrialised countries with regard to their internal situation (economic crisis and growing hostility towards foreigners) and the international situation within the political and economic world system.

Taking as an example the complex case of the Indochinese refugees, the dilemma encountered by the international community is manifested in the absence of a consensual solution with regard to the perpetuation and the destabilizing effects of the refugee problem on the recipient countries.

Humanitarian aid, considered to be politically neutral, suffers from fundamental constraints, and so does development aid which would be necessary to permit refugees to return to a life of self-determination. 